\title{
Coherence versus reliability of stochastic oscillators with delayed feedback
}

\author{
Denis S. Goldobin \\ Department of Theoretical Physics, Perm State University, 15 Bukireva str., 614990, Perm, Russia
}

\begin{abstract}
For noisy self-sustained oscillators, both reliability, stability of a response to a noisy driving, and coherence understood in the sense of constancy of oscillation frequency belong to the main characteristics. Though the both characteristics and techniques for controlling them received great attention of researchers, owing to their importance for neurons, lasers, clocks, electric generators, etc., these characteristics were previously considered separately. In this paper, strong quantitative relation between coherence and reliability is revealed for a limit cycle oscillator subject to a weak noisy driving and a linear delayed feedback, a convectional control tool. Analytical findings are verified and enriched with a numerical simulation for the Van der Pol-Duffing oscillator.
\end{abstract}

PACS numbers: 05.40.-a, 02.50.Ey, 05.45.Xt

Recently, robustness of response of a limit cycle oscillator to a noisy driving have attracted considerable attention of both experimentalists and theoreticians [1, 2, 3, 4, [5, 6, 7, 8, 9, 10]. In different fields of science, related phenomena appear under different names. In neurophysiology the reliability property of spiking neurons, which manifests itself as a coincidence of responses of a single neuron to a repeated noisy input of a prerecorded waveform, attracts great attention [1]. In recent experiments with a noise-driven Nd:YAG (neodymium-doped yttrium aluminum garnet) laser [2], a similar property has been referred as consistency. From the theoretical viewpoint, reliability and consistency are manifestations of the synchronization of uncoupled nonlinear oscillators receiving identical noisy driving [3, 4, 5, 6, 6, 8, 8, 9, 10].

Quantitatively, stability of response, reliability, is characterized by the largest Lyapunov exponent (LE). For smooth limit cycle oscillators LE is negative [3, 5, 7] meaning that the system is reliable. However, a large noise may lead to a positive $\operatorname{LE}([3,6,6,7,[1]$; and even antireliability for neuron-like systems in a "classic" experimental set-up has been forecasted [9]).

However, for some oscillatory systems not only the response stability is important, but also the coherence, i.e., the constancy of the oscillation frequency, which is measured by the diffusion constant of the oscillation phase. The coherence determines the precision of clocks (including biological ones [12]), the quality of electric generators, susceptibility of an oscillatory system to external driving [13], and predisposition to synchronization; a laser radiation should be coherent when one needs to focus the beam or redirect it without angular divergence; etc. In Ref. 13] (followed by methodologically closely related Ref. [14]) the extremely efficient technique for controlling the coherence by a weak delayed feedback has been proposed and theoretically analyzed (a successful experimental implementation of this technique for a laser in chaotic regime has been reported in Ref. [15]). Remarkably, due to the time-shift symmetry a noiseless limit cycle system is neutrally stable and remains such in the presence of a delayed feedback. But in the presence of noise, the delayed feedback utilized for controlling the coherence may considerably affect the response stability.
Noteworthy, in the presence of both delay and noise (or irregularities), the process is not Markovian anymore; therefore, one may not apply such well-elaborated tools as the conventional Fokker-Planck equation, and ad hoc statistical methods are employed for studies [16, 17, 18, 19]. This paper presents both analytical and numerical results on the reliability of noisy-driven limit cycle oscillators subject to delayed feedback control, suggesting an effective mean for controlling the reliability. Analysis of these results in the context of controlling coherence reveals strong quantitative relations [Eq. (13)] between the reliability and the coherence. The disclosed fact, that a high reliability occurs for a weak coherence, and vice versa the weaker reliability the higher coherence, imposes important limitations on implementation of this conventional control technique. Imperfect cases are also discussed.

In order to demonstrate numerically the relationship between coherence and reliability, a simulation for noisy Van der Pol oscillator

$$
\begin{gathered}
\ddot{x}-\mu\left(1-x^{2}\right) \dot{x}+x=k[\dot{x}(t-\tau)-\dot{x}(t)]+\varepsilon \xi(t), \\
\left\langle\xi\left(t_{1}\right) \xi\left(t_{2}\right)\right\rangle=2 \delta\left(t_{1}-t_{2}\right), \quad\langle\xi\rangle=0
\end{gathered}
$$

has been performed. Here $\mu$ describes closeness to the Hopf bifurcation point, $k$ and $\tau$ are the feedback strength and delay time, respectively, $\varepsilon$ is the noise amplitude, $\xi(t)$ is normalized white Gaussian noise. In the presence of noise the oscillation phase $\varphi=-\arctan (x / \dot{x})$ diffuses according to $\left\langle(\varphi(t)-\langle\varphi(t)\rangle)^{2}\right\rangle \propto D t$. Diffusion constant $D$ quantifies the coherence of oscillations.

Fig. 1 shows the effects of a linear delayed feedback on the diffusion constant (DC) and the Lyapunov exponent (LE) measuring exponential growth rate of perturbations in the system (11). Noteworthy, not merely the LE and the DC are crucially magnified or suppressed simultaneously at $\tau / T_{0}$ (here $T_{0}$ is the oscillation period of the controlfree noiseless system) being integers and half-integers, but even their ratio remains nearly constant as $\tau$ changes (see Fig.10).

Let us develop a phase description of the system. One can parameterize the states of a limit cycle system on the limit cycle by the oscillation phase $\varphi$ uniformly grow- 

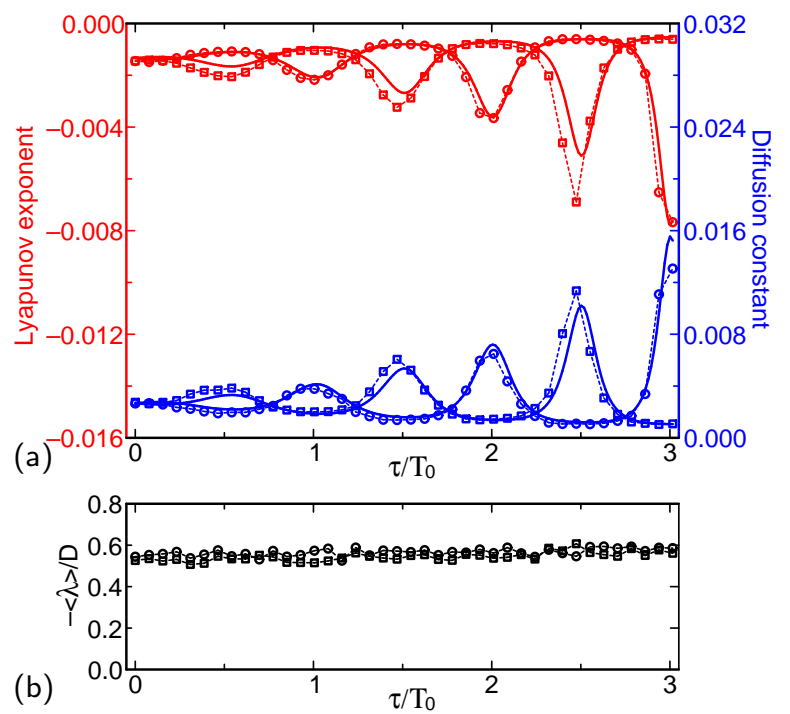

FIG. 1: (Color online) Dependencies of Lyapunov exponent $\langle\lambda\rangle$ (a: upper graphs) and diffusion constant $D$ (a: lower graphs) on delay time $\tau$ for the Van der Pol oscillator (1) with $\mu=0.7$ subject to white Gaussian noise of strength $\varepsilon^{2}=0.01$ and the linear delayed feedback of strength $k=0.06$ (squares) and $k=-0.06$ (circles). Oscillation period of the control-free noiseless system $T_{0} \approx 2 \pi / 0.96$. The solid lines present analytical dependencies [Eqs. (11), (12)]. (b): The inconstancy of ratio $-\langle\lambda\rangle / D$ is not resolvable against the background of the calculation inaccuracy.

ing in the course of temporal evolution. Such an oscillator subject to weak noise and feedback stays in the vicinity of this cycle, and its evolution may be still described within the framework of the conventional phase approximation [20]. Close to the bifurcation point, i.e., for $\mu \rightarrow 0$, the Van der Pol oscillator has a nearly circular limit cycle: $x_{0}=2 \cos \varphi, \dot{x}_{0}=-2 \sin \varphi$, and the phase equation for the system (11) reads (cf. [13])

$$
\dot{\varphi}=\Omega_{0}+a g[\varphi(t-\tau), \varphi(t)]+\varepsilon f[\varphi(t)] \circ \xi(t),
$$

where $\Omega_{0}=2 \pi / T_{0}$ is the inherent cyclic frequency of the system, $a=k / 2, g=\sin [\varphi(t-\tau)-\varphi(t)]$, the sign "o" means a Stratonovich form of the equation, $2 \pi$-periodic function $f(\varphi)$ is the sensitivity to noise. For an additive noise as in Eq. (11), $f(\varphi)=\left(2 \Omega_{0}\right)^{-1} \cos \varphi$ (cf. 13]), but we keep $f$ for generality. Noteworthy, in Ref. [18], Eq. (2) has been used to describe the evolution of the phase of an optical field in a laser with a weak optical feedback.

For a small perturbation $\alpha$, one finds

$$
\begin{aligned}
\dot{\alpha}=a \cos [\varphi(t-\tau)-\varphi(t)] & (\alpha(t-\tau)-\alpha(t)) \\
& +\varepsilon f^{\prime}[\varphi(t)] \alpha(t) \circ \xi(t)
\end{aligned}
$$

(the prime stands for derivative with respect to the argument). Therefore, the instant exponential growth rate $\lambda(t)$ obeys

$$
\begin{array}{r}
\lambda(t)=a \cos [\varphi(t-\tau)-\varphi(t)]\left(e^{-\int_{t-\tau}^{t} \lambda\left(t_{1}\right) \mathrm{d} t_{1}}-1\right) \\
+\varepsilon f^{\prime}[\varphi(t)] \circ \xi(t),
\end{array}
$$
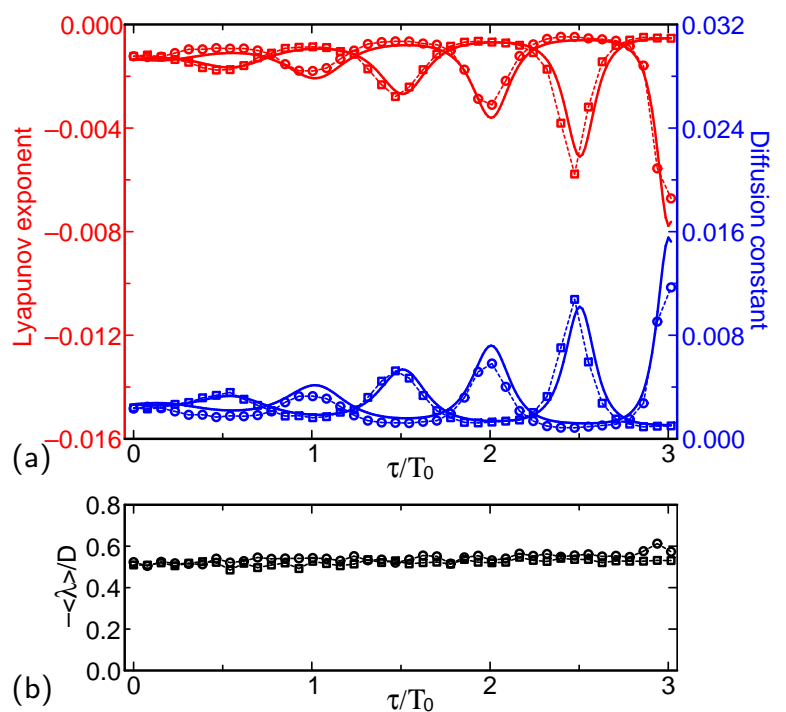

FIG. 2: (Color online) Same dependencies as in Fig.1 for the same parameter values but for red Gaussian noise $\zeta(t)=$ $T^{-1} \int_{t-T}^{t} \xi\left(t_{1}\right) \mathrm{d} t_{1}$ with $T=1.2$. For notation see caption to Fig.1

here we have made use of $\alpha(t) \propto \exp \left(\int^{t} \lambda\left(t_{1}\right) \mathrm{d} t_{1}\right)$. Note, that the $L E$ is the mean value $\langle\lambda\rangle$.

For further analysis, it is more convenient to consider equations in Ito form. Eqs. (2), (3) read

$$
\begin{array}{r}
\dot{\varphi}=\Omega_{0}+a \sin [\varphi(t-\tau)-\varphi(t)]+\varepsilon^{2} f^{\prime} f+\varepsilon f[\varphi(t)] \xi(t), \\
\lambda(t)=a \cos [\varphi(t-\tau)-\varphi(t)]\left(e^{-\int_{t-\tau}^{t} \lambda\left(t_{1}\right) \mathrm{d} t_{1}}-1\right) \\
+\varepsilon^{2} f^{\prime \prime} f+\varepsilon f^{\prime}[\varphi(t)] \xi(t) .
\end{array}
$$

The terms ahead of the noisy ones describe the Stratonovich drift. Recall, in Ito form (with the Stratonovich drift included explicitly) the instant value $\varphi(t)$ is independent of the instant value $\xi(t)$ taken at the same time moment $t$.

Let us explicitly introduce the mean frequency $\Omega$ and the instant frequency deviation $v ; \varphi \equiv \Omega t+\psi, \dot{\psi}=v$, $\langle v\rangle=0$. For a weak noise and a small feedback strength $(\varepsilon \ll 1,|a| \ll 1)$ the instant frequency fluctuations are small $(v \ll 1)$, and Eqs. (44),(5) yield, up to the main order of accuracy,

$$
\begin{array}{rl}
\Omega= & \Omega_{0}-a \sin \Omega \tau, \\
v= & -a \cos \Omega \tau[\psi(t-\tau)-\psi(t)]+\varepsilon^{2} f^{\prime} f+\varepsilon f \xi(t), \\
\lambda=a & a(\cos \Omega \tau+\sin \Omega \tau[\psi(t-\tau)-\psi(t)]) \\
& \times\left(e^{-\int_{t-\tau}^{t} \lambda\left(t_{1}\right) \mathrm{d} t_{1}}-1\right)+\varepsilon^{2} f^{\prime \prime} f+\varepsilon f^{\prime} \xi(t) .
\end{array}
$$

Let us now find the LE. Assuming $v$ and the fluctuating part $\widetilde{\lambda}$ of $\lambda$, obeying

$$
\widetilde{\lambda}(t) \approx-a \cos \Omega \tau \int_{t-\tau}^{t} \widetilde{\lambda}\left(t_{1}\right) \mathrm{d} t_{1}+\varepsilon f^{\prime}(\Omega t) \xi(t),
$$

to be Gaussian, one can employ Furutsu-Novikov for- 
(a)

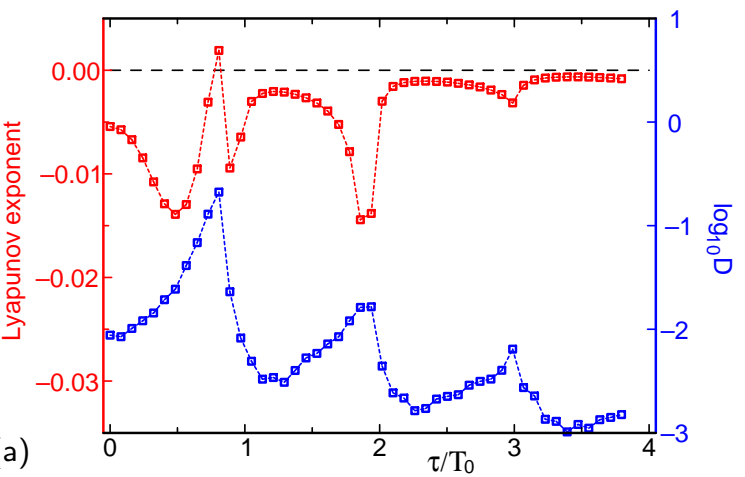

(b)

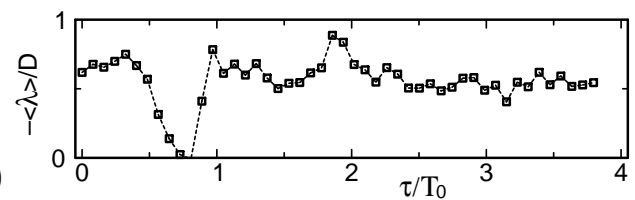

FIG. 3: (Color online) Dependencies $\langle\lambda\rangle(\tau)$ (a: upper graph) and $D(\tau)$ (a: lower graph) for the Van der Pol-Duffing oscillator (14) with $\mu=0.2\left(T_{0} \approx 2 \pi / 2.02\right)$ subject to white Gaussian noise, $\varepsilon=0.05$, and the delayed feedback, $k=0.06$. For description and notation of (b), see Fig.1 caption.

mula [21] to obtain from Eq. (8) [23]

$\langle\lambda\rangle=a \cos \Omega \tau\left(-\langle\lambda\rangle \tau+\frac{1}{2}\left\langle\left[\int_{t-\tau}^{t} \widetilde{\lambda}\left(t_{1}\right) \mathrm{d} t_{1}\right]^{2}\right\rangle\right)-\varepsilon^{2}\left\langle f^{\prime 2}\right\rangle_{\varphi}$

(here $\langle\ldots\rangle_{\varphi}$ stands for an average over the phase $\varphi$ ). The value $I \equiv\left\langle\left[\int_{t-\tau}^{t} \widetilde{\lambda}\left(t_{1}\right) \mathrm{d} t_{1}\right]^{2}\right\rangle$ can be evaluated from Eq. (9) (similarly to $\left\langle v\left(t_{1}\right) v\left(t_{2}\right)\right\rangle$ in Ref. [13]);

$$
\begin{aligned}
I & =\varepsilon^{2}\left\langle f^{\prime 2}\right\rangle_{\varphi} \frac{\tau}{\pi} \int_{-\infty}^{+\infty}\left|\frac{i x}{1-e^{-i x}}+a \tau \cos \Omega \tau\right|^{-2} \mathrm{~d} x \\
& =\varepsilon^{2}\left\langle f^{\prime 2}\right\rangle_{\varphi}\left\{\begin{array}{c}
\frac{2 \tau}{1+a \tau \cos \Omega \tau}, \quad \text { for } a \tau \cos \Omega \tau>-1 ; \\
\frac{2 \tau}{\pi}\left[\frac{2}{1+a \tau \cos \Omega \tau}\right]^{\frac{8}{7}}, \text { for } a \tau \cos \Omega \tau<-1 .
\end{array}\right.
\end{aligned}
$$

For $a \tau>1$, Eq. (6) exhibits multistability of mean frequency $\Omega[13,17,18]$, which results in the violation of the basic assumptions of our analytical theory. Hence, the case $a \tau \cos \Omega \tau<-1$ may be ignored as meaningless, and after substitution of $I$, Eq. (10) reads

$$
\langle\lambda\rangle=-\frac{\varepsilon^{2}\left\langle f^{\prime 2}\right\rangle_{\varphi}}{(1+a \tau \cos \Omega \tau)^{2}},
$$

whereas the DC has been already evaluated in Ref. [13];

$$
D=\frac{2 \varepsilon^{2}\left\langle f^{2}\right\rangle_{\varphi}}{(1+a \tau \cos \Omega \tau)^{2}} .
$$

Therefore,

$$
-\frac{\langle\lambda\rangle}{D}=\frac{\left\langle f^{\prime 2}\right\rangle_{\varphi}}{2\left\langle f^{2}\right\rangle_{\varphi}}=\text { const }
$$

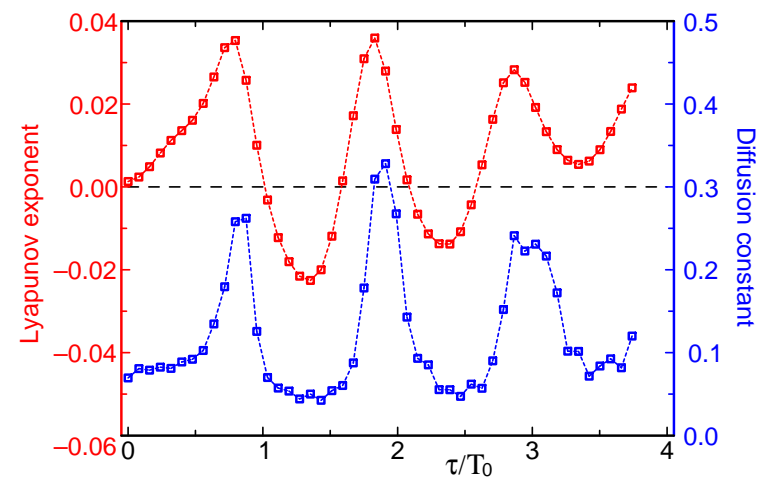

FIG. 4: (Color online) Same dependencies as in Fig. 3a for the same Van der Pol-Duffing system and feedback strength but a larger noise, $\varepsilon=0.374$.

which is $1 / 2$ for $f(\varphi)=\left(2 \Omega_{0}\right)^{-1} \cos \varphi$ as for the Van der Pol system in Fig. 1. Note that, due to the deformation of the limit cycle at $\mu=0.7$, relation (13) is more accurate than Eqs. (11), (12) where the term $a \tau \cos \Omega \tau$ is specific to $g=\sin [\varphi(t-\tau)-\varphi(t)]$ (see Fig.11 where $-\langle\lambda\rangle / D \approx 0.55$ ).

While deriving Eqs. (11), (12) we nowhere utilized that the noise is $\delta$-correlated. Remarkably, the results remain valid for colored noise, e.g., a red one (see Fig.21), results for which coincide with the one for white noise almost up to the numerical calculation inaccuracy and $-\left\langle\lambda_{\text {red }}\right\rangle / D_{\text {red }} \approx 0.53$.

For a strong noise the phase description always leading to a negative LE is not applicable, and even positive LEs have been reported [3, 6, 7, 8, 9]. This case can be treated only numerically. For this sake, a simulation for the Van der Pol-Duffing oscillator

$$
\ddot{x}-\mu\left(1-x^{2}\right) \dot{x}+x+x^{3}=k[\dot{x}(t-\tau)-\dot{x}(t)]+\varepsilon \xi(t)
$$

exhibiting positive LEs for a moderate noise [6] has been performed. Let us note that for a non-large noise (Fig.[3), ratio $-\langle\lambda\rangle / D$ is changed not greater than by $20 \%$, while the DC and the LE are changed by factor $\approx 20$, in a broad range of $\tau$ (the only exception is interval $\left[T_{0} / 2, T_{0}\right]$ near the domain where the LE is positive).

At $\varepsilon=0.374$ (Fig.4), where the control-free Van der Pol-Duffing oscillator just becomes unstable (unreliable),

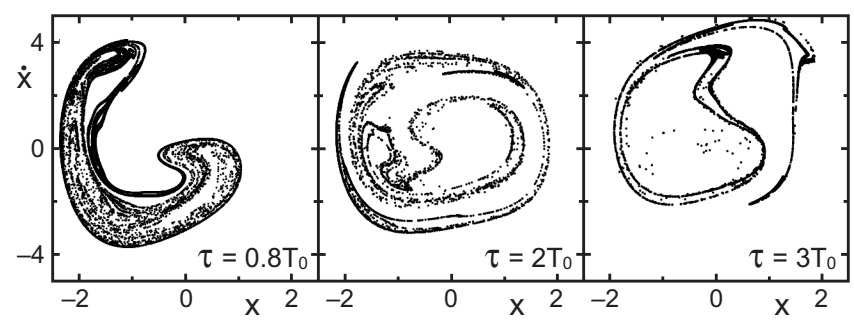

FIG. 5: The snapshots of the ensemble of 20000 identical Van der Pol-Duffing oscillators $(\mu=0.2)$ driven by common white Gaussian noise $(\varepsilon=0.374)$ have a fine structure for $\tau$ indicated in the plots (cf. to the LE in Fig.4). 
$\left.\langle\lambda\rangle\right|_{\tau=0} \approx 0$, the linear delayed feedback leads to maximal positive LEs for integer $\tau / T_{0}$ and minimal (not always negative) LEs for half-integer $\tau / T_{0}$. Concerning the interpretation of dependence $\langle\lambda\rangle(\tau)$, note the following. In the absence of the feedback control, an intermittency of epochs of positive and negative local LEs ("local" means evaluated over a finite time interval) takes place and the transition to positive LE is related to a plain quantitative prevalence of the former over the latter (cf. [9] ). The feedback affects (magnifies or suppresses) the local LEs over these epochs non-uniformly, thus shifting balance between these epoches and bringing about a domination of positive local LEs for integer $\tau / T_{0}$ and negative ones for half-integer $\tau / T_{0}$. For positive "global" LEs, phase diffusion owes mainly not to stochasticity but to chaos (samples of snapshot chaotic attractors, see Ref. [22], are presented in Fig. (5). As a result, here the DC is diminished where the LE is minimal.

Summarizing, for a weak white or colored Gaussian noise [24], highly stable response (reliability) to a noisy driving is observed when phase diffusion is strong (i.e., the coherence is weak). Vice versa, for small diffusion (i.e., highly coherent oscillations) response is weakly stable [Figs. 1,2, Eq. (13)]. In particular, this imposes strong limitations on the implementation of the technique of coherence improvement by virtue of a linear delayed feed- back. For instance, in an ensemble of uncoupled identical self-sustained oscillators synchronized by a common external noisy driving, small intrinsic noise is always present and leads to spreading of oscillator phases: $\Delta \varphi \propto \varepsilon_{\text {in }} / \sqrt{-\langle\lambda\rangle}\left(\varepsilon_{\text {in }}\right.$ is the amplitude of intrinsic noise, cf. [6]). In such an ensemble the delayed feedback improvement of the coherence results in a mutual spreading of oscillator phases which may be sometimes undesirable.

For a strong noise being capable to create a positive Lyapunov exponent, i.e., antireliability, chaotic contribution to phase diffusion may prevail over the stochastic one, and then an enhanced coherence occurs for the maximal reliability (Fig. (4).

Detailed calculation of the Lyapunov exponent in the above text serves the purpose to disclose the essentially different nature of various contributions to the Lyapunov exponent and the diffusion constant. However, the final quantitative effect of delayed feedback on these dissimilar properties of oscillatory systems somewhat surprisingly turns out to be identical. The reported phenomenon being valid for a general class of limit cycle oscillators is, thus, neither intuitively expected nor trivial.

I thank A. Pikovsky and M. Zaks for useful discussions and appreciate financial support from the Foundation "Perm Hydrodynamics," CRDF (Grant No. Y5P0901), and MESRF (Grant No. 2.2.2.3.16038).
[1] Z. F. Mainen and T. J. Sejnowski, Science 268, 1503 (1995); C. V. Rao, D. M. Wolf, and A. P. Arkin, Nature 420, 231 (2002).

[2] A. Uchida, R. McAllister, and R. Roy, Phys. Rev. Lett. 93, 244102 (2004)

[3] A. S. Pikovsky, Radiophys. Quantum Electron. 27, 576 (1984).

[4] L. Yu, E. Ott, and Q. Chen, Phys. Rev. Lett. 65, 2935 (1990); A. S. Pikovsky, Phys. Lett. A 165, 33 (1992); P. Khoury, M. A. Lieberman, and A. J. Lichtenberg, Phys. Rev. E 54, 3377 (1996); J. Ritt, ibid. 68, 041915 (2003).

[5] J. N. Teramae and D. Tanaka, Phys. Rev. Lett. 93, 204103 (2004); D. S. Goldobin and A. S. Pikovsky, Physica A 351, 126 (2005).

[6] D. S. Goldobin and A. Pikovsky, Phys. Rev. E 71, 045201(R) (2005).

[7] K. Nagai, H. Nakao, and Y. Tsubo, Phys. Rev. E 71, 036217 (2005); H. Nakao, K. Nagai, and K. Arai, Prog. Theor. Phys. Suppl. 161, 294 (2006).

[8] H. Nakao et al., Phys. Rev. E 72, 026220 (2005); K. Arai and H. Nakao, ibid. 77, 036218 (2008).

[9] D. S. Goldobin and A. Pikovsky, Phys. Rev. E 73, 061906 (2006).

[10] H. Nakao, K. Arai, and Y. Kawamura, Phys. Rev. Lett. 98, 184101 (2007).

[11] L. Schimansky-Geier and H. Herzel, J. Stat. Phys. 70, 141 (1993).

[12] M. Montminy, Annu. Rev. Biochem. 66, 807 (1997); D. Bell-Pedersen et al., Nat. Rev. Genet. 6, 544 (2005).

[13] D. Goldobin, M. Rosenblum, and A. Pikovsky, Phys. Rev.
E 67, 061119 (2003); Physica A 327, 124 (2003).

[14] A. H. Pawlik and A. Pikovsky, Phys. Lett. A 358, 181 (2006); N. Tukhlina, M. Rosenblum, and A. Pikovsky, Physica A 387, 6045 (2008).

[15] S. Boccaletti, E. Allaria, and R. Meucci, Phys. Rev. E 69, 066211 (2004).

[16] L. S. Tsimring and A. Pikovsky, Phys. Rev. Lett. 87, 250602 (2001); S. Guillouzic, I. L'Heureux, and A. Longtin, Phys. Rev. E 59, 3970 (1999); T. Ohira and T. Yamane, ibid. 61, 1247 (2000).

[17] E. Niebur, H. G. Schuster, and D. M. Kammen, Phys. Rev. Lett. 67, 2753 (1991).

[18] C. Masoller, Phys. Rev. Lett. 88, 034102 (2002).

[19] T. D. Frank and P. J. Beek, Phys. Rev. E 64, 021917 (2001); T. D. Frank, ibid. 66, 011914 (2002); ibid. 69, 061104 (2004); ibid. 71, 031106 (2005); ibid. 72, 011112 (2005).

[20] Y. Kuramoto, Chemical Oscillations, Waves and Turbulence (Dover, New York, 2003).

[21] K. Furutsu, J. Res. Natl. Bur. Stand., Sect. D 67, 303 (1963); Y. Novikov, Zh. Eksp. Teor. Fiz. 47, 1919 (1964) [Sov. Phys. JETP 20, 1290 (1965)].

[22] L. Yu, Q. Chen, and E. Ott, Physica D 53, 102 (1991).

[23] Notice, Eq. (8) should not be linearized with respect to $\lambda$.

[24] Perhaps, the diffusionless noises as a blue one should be excluded because for them, unlikely to the case studied, phase diffusion would be purely due to an interaction between the noise and the system nonlinearity. 